\title{
POSITIVITY AND PERIODICITY OF SOLUTIONS FOR FIRST-ORDER NEUTRAL DELAY DYNAMIC EQUATIONS
}

\author{
KAMEL ALI KHELIL ${ }^{1}$, ABDELOUAHEB ARDJOUNI ${ }^{2, *}$, AHCENE DJOUDI ${ }^{3}$ \\ ${ }^{1}$ Laboratory of Analysis and Control of Differential Equations "ACED", University of Guelma, Algeria \\ ${ }^{2}$ Department of Mathematics and Informatics, University of Souk Ahras, Algeria \\ ${ }^{3}$ Department of Mathematics, University of Annaba, Algeria \\ *Corresponding author: abd_ardjouni@yahoo.fr
}

Received Dec. 20, 2020

АвSTRACт. In this work, we use the Krasnoselskii theorem to study the positivity and periodicity of solutions for the following first-order neutral delay dynamic equation on time scales

$$
\begin{aligned}
& \left(r(t)\left[x(t)-P(t) \int_{c}^{d} x(t-\tau(t, \xi)) \Delta \xi\right]\right)^{\Delta} \\
& =-Q(t) x^{\sigma}(t)+\int_{c}^{d} f(t, x(t-\tau(t, \xi))) \Delta \xi .
\end{aligned}
$$

The new results obtained in this paper extend the results in the literature.

2010 Mathematics Subject Classification. 34K20; 34N05; 45J05; 45D05.

Key words and phrases. Periodic solutions; Neutral dynamic equations; Time scales; Fixed point theorem.

\section{INTRODUCTION}

The study of neutral dynamic equations has many applications in various areas of science and engineering. Many researchers have studied the existence and periodicity of this equations using fixed points theorem. Some new results for the discrete and continuous cases have been given by studying the more general time scales case (see [1]- [5], [9], [13]).

Time scale theory was introduced for the first time by Hilger [12] in 1988 to unify discrete and continuous analysis. However, some physical systems are modeled by so-called dynamic equations because they are differential equations, difference equations or a combination of 
both. Therefore, the calculation of time scales provides a generalization of the differential and difference analysis.

In [10] Candan has used the fixed point method to study the positivity and periodicity of solutions for the neutral delay differential equation having the form

$$
\begin{aligned}
& \left(r(t)\left[x(t)-P(t) \int_{c}^{d} x(t-\tau(t, \xi)) d \xi\right]\right)^{\prime} \\
& =-Q(t) x(t)+\int_{c}^{d} f(t, x(t-\tau(t, \xi))) d \xi .
\end{aligned}
$$

Let $\mathbb{T}$ be a periodic time scale with $0 \in \mathbb{T}$. The aim of this work is to extend the results obtained in [10] to the first-order neutral delay dynamic equation on a time scale. More precisely, we consider the equation

$$
\begin{aligned}
& \left(r(t)\left[x(t)-P(t) \int_{c}^{d} x(t-\tau(t, \xi)) \Delta \xi\right]\right)^{\Delta} \\
& =-Q(t) x^{\sigma}(t)+\int_{c}^{d} f(t, x(t-\tau(t, \xi))) \Delta \xi,
\end{aligned}
$$

where $r \in C_{r d}^{1}(\mathbb{T}, \mathbb{R}), r(t)>0, \tau \in C_{r d}\left(\mathbb{T} \times[c, d]_{\mathbb{T}},(0, \infty)_{\mathbb{T}}\right), Q \in C_{r d}(\mathbb{T},(0, \infty)), P \in$ $C_{r d}(\mathbb{T}, \mathbb{R}), f \in C_{r d}(\mathbb{T} \times \mathbb{R}, \mathbb{R}), d>c \geq 0$, and $r, P, Q$ are $\omega$-periodic functions, $f$ is $\omega$-periodic with respect to first variable, and $\tau$ is $\omega$-periodic with respect to $t$.

\section{Preliminaries}

Definition 2.1. A time scale $\mathbb{T}$ is an arbitrary nonempty closed subset of the real numbers $\mathbb{R}$.

Since we are interested in periodic solutions, we suppose that the time scale under consideration is not bounded above and below. Throughout this paper, intervals subscripted with a $\mathbb{T}$ represent real intervals intersected with $\mathbb{T}$. For example, $a, b \in \mathbb{T},[a, b]_{\mathbb{T}}=[a, b] \cap \mathbb{T}$.

We begin this section by considering some advanced topics in the theory of dynamic equations on time scales. Most of the following definitions, lemmas and theorems can be found in $[7,8]$.

Definition 2.2. The forward and backward jump operators $\sigma, \rho: \mathbb{T} \rightarrow \mathbb{T}$ and the graininess function $\mu: \mathbb{T} \rightarrow[0, \infty)$ are defined, respectively, by

$$
\sigma(t)=\inf \{s \in \mathbb{T}: s>t\}, \rho(t)=\sup \{s \in \mathbb{T}: s<t\}, \mu(t)=\sigma(t)-t .
$$

We make the assumption that $\inf \emptyset=\sup \mathbb{T}$ and $\sup \emptyset=\inf \mathbb{T}$. A point $t \in \mathbb{T}$ is called rightdense if $t<\sup \mathbb{T}$ and $\sigma(t)=t$, right-scattered if $\sigma(t)>t$, left-dense if $t>\inf \mathbb{T}$ and $\rho(t)=t$, 
and left-scattered if $\rho(t)<t$. If $\mathbb{T}$ has a left-scattered maximum $m$, define $\mathbb{T}^{k}=\mathbb{T}-\{m\}$. Otherwise, $\mathbb{T}^{k}=\mathbb{T}$. Finally, if $f: \mathbb{T} \rightarrow \mathbb{R}$ we define the function $f^{\sigma}: \mathbb{T} \rightarrow \mathbb{R}$ by

$$
f^{\sigma}(t)=f(\sigma(t)) \text { for all } t \in \mathbb{T}
$$

Definition 2.3. A function $f: \mathbb{T} \rightarrow \mathbb{R}$ is called $r d$-continuous provided it is continuous at every right-dense point $t \in \mathbb{T}$ and its left-sided limits exist, and is finite at every left-dense point $t \in \mathbb{T}$. The set of $r d$-continuous functions $f: \mathbb{T} \rightarrow \mathbb{R}$ will be denoted by

$$
C_{r d}=C_{r d}(\mathbb{T})=C_{r d}(\mathbb{T}, \mathbb{R})
$$

The set of functions $f: \mathbb{T} \rightarrow \mathbb{R}$ that are differentiable and whose derivative is $r d$-continuous is denoted by

$$
C_{r d}^{1}=C_{r d}^{1}(\mathbb{T})=C_{r d}^{1}(\mathbb{T}, \mathbb{R})
$$

Definition 2.4. For $f: \mathbb{T} \rightarrow \mathbb{R}$, we define $f^{\Delta}(t)$ to be the number (if it exists) with the property that for any given $\varepsilon>0$, there exists a neighborhood $U$ of $t$ such that

$$
\left|(f(\sigma(t))-f(s))-f^{\Delta}(t)(\sigma(t)-s)\right|<\varepsilon|\sigma(t)-s| \text { for all } s \in U
$$

The function $f^{\Delta}: \mathbb{T}^{k} \rightarrow \mathbb{R}$ is called the delta (or Hilger) derivative of $f$ on $\mathbb{T}^{k}$.

Theorem 2.1. Suppose $f: \mathbb{T} \rightarrow \mathbb{R}$ is a function and let $t \in \mathbb{T}^{k}$. Then, we have the following,

(i) if $f$ is differentiable at $t$, then $f$ is continuous at $t$,

(ii) if $f$ is continuous at $t$ and $t$ is right-scattered, then $f$ is differentiable at $t$ with

$$
f^{\Delta}(t)=\frac{f(\sigma(t))-f(t)}{\mu(t)},
$$

(iii) if $t$ is right-dense, then $f$ is differentiable at $t$ with

$$
f^{\Delta}(t)=\lim _{s \rightarrow t} \frac{f(t)-f(s)}{t-s} .
$$

Theorem 2.2. Suppose $f, g: \mathbb{T} \rightarrow \mathbb{R}$ are differentiable at $t \in \mathbb{T}^{k}$. Then

(i) $(f+g)^{\Delta}(t)=f^{\Delta}(t)+g^{\Delta}(t)$.

(ii) The derivative of product $f g: \mathbb{T} \rightarrow \mathbb{R}$ is given by

$$
(f g)^{\Delta}=f^{\Delta}(t) g(t)+f^{\sigma}(t) g^{\Delta}(t)=f(t) g^{\Delta}(t)+f^{\Delta}(t) g^{\sigma}(t) .
$$

(iii) If $f(t) f^{\sigma}(t) \neq 0$, then $\frac{1}{f}$ is differentiable at $t$ with

$$
\left(\frac{1}{f}\right)^{\Delta}(t)=-\frac{f^{\Delta}(t)}{f(t) f^{\sigma}(t)}
$$


Definition 2.5. For $a, b \in \mathbb{T}$, the Cauchy integral of $f^{\Delta}$ is defined by

$$
\int_{a}^{b} f^{\Delta}(t) \Delta t=f(b)-f(a)
$$

and infinite integrals are defined as

$$
\int_{a}^{\infty} f(s) \Delta s=\lim _{t \rightarrow \infty} \int_{a}^{t} f(s) \Delta s .
$$

Definition 2.6. A function $p: \mathbb{T} \rightarrow \mathbb{R}$ is called regressive provided $1+\mu(t) p(t) \neq 0$ for all $t \in \mathbb{T}$. The set of all regressive and $r d$-continuous functions $p: \mathbb{T} \rightarrow \mathbb{R}$ will be denoted by $\mathcal{R}=\mathcal{R}(\mathbb{T}, \mathbb{R})$. We define the set $\mathcal{R}^{+}$of all positively regressive elements of $\mathcal{R}$ by

$$
\mathcal{R}^{+}=\mathcal{R}^{+}(\mathbb{T}, \mathbb{R})=\{p \in \mathcal{R}: 1+\mu(t) p(t)>0, \forall t \in \mathbb{T}\}
$$

Definition 2.7 ( [14]). We say that a time scale $\mathbb{T}$ is periodic if there exists $T>0$ such that if $t \in \mathbb{T}$ then $t \pm T \in \mathbb{T}$. For $\mathbb{T} \neq \mathbb{R}$, the smallest positive $T$ is called the period of the time scale.

Remark 2.1 ( [14]). All periodic time scales are unbounded above and below.

Definition 2.8 ( $[14])$. Let $\mathbb{T} \neq \mathbb{R}$ be a periodic time scale with period $T$. We say that the function $f: \mathbb{T} \rightarrow \mathbb{R}$ is periodic with period $\omega$ if there exists a natural number $n$ such that $\omega=n T, f(t+\omega)=$ $f(t)$ for all $t \in \mathbb{T}$ and $\omega$ is the smallest number such that $f(t+\omega)=f(t)$.

If $\mathbb{T}=\mathbb{R}$, we say that $f$ is periodic with period $\omega>0$ if $\omega$ is the smallest positive number such that $f(t+\omega)=f(t)$ for all $t \in \mathbb{T}$.

Remark 2.2 ( [14]). If $\mathbb{T}$ is a periodic time scale with period $T$, then $\sigma(t+n T)=\sigma(t)+n T$. Consequently, the graininess function $\mu$ satisfies $\mu(t+n T)=\mu(t)$ and so is a periodic function with period $T$.

Definition $2.9([7])$. Let $p \in \mathcal{R}$, then the generalized exponential function $e_{p}$ is defined as the unique solution of the initial value problem

$$
x^{\Delta}(t)=p(t) x(t), x(s)=1, \text { where } s \in \mathbb{T} .
$$

An explicit formula for $e_{p}(t, s)$ is given by

$$
e_{p}(t, s)=\exp \left(\int_{s}^{t} \zeta_{\mu(\tau)}(p(\tau)) \Delta \tau\right), \text { for all } s, t \in \mathbb{T},
$$

with

$$
\zeta_{h}(\tau)= \begin{cases}\frac{\log (1+h \tau)}{h} & \text { if } h \neq 0 \\ \tau & \text { if } h=0\end{cases}
$$

where log is the principal logarithm function. 
Lemma 2.1 ( [7]). Let $p \in \mathcal{R}$, then

(i) $e_{0}(t, s)=1$ and $e_{p}(t, t)=1$,

(ii) $e_{p}(\sigma(t), s)=(1+\mu(t) p(t)) e_{p}(t, s)$,

(iii) $e_{p}^{\Delta}(t, s)=p(t) e_{p}(t, s)$,

(iv) $\frac{1}{e_{p}(t, s)}=e_{\ominus p}(t, s)$ with $\ominus p=-\frac{p}{1+\mu p}$,

$(v) e_{p}(t, s)=\frac{1}{e_{p}(s, t)}=e_{\ominus p}(s, t)$,

$(v i) e_{p}(t, s) e_{p}(s, r)=e_{p}(t, r)$.

Lemma $2.2([7])$. If $p \in \mathcal{R}^{+}$, then

$$
0<e_{p}(t, s) \leq \exp \left(\int_{s}^{t} p(\tau) \Delta \tau\right)
$$

for all $t \in[s, \infty)_{\mathbb{T}}$.

Theorem 2.3 ( [6]). Let $\mathbb{T}$ be a periodic time scale with period $T>0$. If $p \in C_{r d}(\mathbb{T})$ is a periodic function with the period $\omega=n T$, then

$$
\begin{gathered}
\int_{a+\omega}^{b+\omega} p(u) \Delta u=\int_{a}^{b} p(u) \Delta u \\
e_{p}(b+\omega, a+\omega)=e_{p}(b, a)
\end{gathered}
$$

and $e_{p}(t+\omega, t)$ is independent of $t \in \mathbb{T}$ whenever $p \in \mathcal{R}$.

\section{Main Results}

Let $\Phi=\left\{x \in C_{r d}(\mathbb{T}, \mathbb{R}), x(t)=x(t+\omega), t \in \mathbb{T}\right\}$ with the sup norm $\|x\|=\sup _{t \in[0, \omega]_{\mathbb{T}}}|x(t)|$. It is clear that $\Phi$ is a Banach space. Let $r_{0}=\min _{t \in[0, \omega]_{\mathbb{T}}} r(t), r_{1}=\max _{t \in[0, \omega]_{\mathbb{T}}} r(t)$ and $\frac{Q(t)}{r^{\sigma}(t)} \in \mathcal{R}^{+}$.

Now, we give the Krasnoselskii fixed point theorem which enables us to show the existence of a positive periodic solution.

Theorem 3.1 ( [15]). Let $\mathbb{M}$ be a bounded closed convex nonempty subset of a Banach space $(\mathbb{B},\|\|$.$) .$ Assume $\mathcal{A}$ and $\mathcal{B}$ map $\mathbb{M}$ into $\mathbb{B}$ such that

(i) $x, y \in \mathbb{M}$ imply $\mathcal{A} x+\mathcal{B} y \in \mathbb{M}$,

(ii) $\mathcal{A}$ is completely continuous,

(iii) $\mathcal{B}$ is a contraction mapping.

Then, there exists $z \in \mathbb{M}$ with $\mathcal{A} z+\mathcal{B} z=z$.

Lemma 3.1 ( [14]). Let $x \in \Phi$. Then $\left\|x^{\sigma}\right\|$ exists and $\left\|x^{\sigma}\right\|=\|x\|$. 
Theorem 3.2. Assume $0 \leq P(t)(d-c) \leq p_{1}<1$ and there exist positive constants $m$ and $M$ with $0<m<M$ such that

$$
\frac{r_{1} m}{(d-c)} \leq r^{\sigma}(t)\left[\frac{f(t, x)}{Q(t)}-P^{\sigma}(t) x^{\sigma}(t)\right] \leq \frac{r_{0}\left(1-p_{1}\right) M}{(d-c)}, \quad \forall(t, x) \in[0, \omega]_{\mathbb{T}} \times[m, M]
$$

Then, (1.1) has a positive $\omega$-periodic solution $x \in[m, M]$.

Proof. We note that finding an $\omega$-periodic solution of

$$
\begin{aligned}
& \left(r(t)\left[x(t)-P(t) \int_{c}^{d} x(t-\tau(t, \xi)) \Delta \xi\right]\right)^{\Delta} \\
& =-Q(t) x^{\sigma}(t)+\int_{c}^{d} f(t, x(t-\tau(t, \xi))) \Delta \xi \\
& =-Q(t)\left[x^{\sigma}(t)-P^{\sigma}(t) \int_{c}^{d} x^{\sigma}(t-\tau(t, \xi)) \Delta \xi\right] \\
& +\int_{c}^{d} f(t, x(t-\tau(t, \xi))) \Delta \xi-Q(t) P^{\sigma}(t) \int_{c}^{d} x^{\sigma}(t-\tau(t, \xi)) \Delta \xi \\
& -A(t)\left(r(t)\left[x(t)-P(t) \int_{c}^{d} x(t-\tau(t, \xi)) \Delta \xi\right]\right)^{\sigma} \\
& +\int_{c}^{d} f(t, x(t-\tau(t, \xi)))-Q(t) P^{\sigma}(t) x^{\sigma}(t-\tau(t, \xi)) \Delta \xi,
\end{aligned}
$$

where

$$
A(t):=\frac{Q(t)}{r^{\sigma}(t)}
$$

Multiplying through (3.2) by $e_{A}(t, 0)$ gives

$$
\begin{aligned}
& {\left[r(t)\left(x(t)-P(t) \int_{c}^{d} x(t-\tau(t, \xi)) \Delta \xi\right) e_{A}(t, 0)\right]^{\Delta}} \\
& =\left[\int_{c}^{d} f(t, x(t-\tau(t, \xi)))-Q(t) P^{\sigma}(t) x^{\sigma}(t-\tau(t, \xi)) \Delta \xi\right] e_{A}(t, 0) .
\end{aligned}
$$

Integrating (3.3) from $t$ to $t+\omega$ gives

$$
\begin{aligned}
x(t) & =\frac{1}{r(t)} \int_{t}^{t+\omega} G(t, s) \int_{c}^{d} f(s, x(s-\tau(s, \xi)))-Q(s) P^{\sigma}(s) x^{\sigma}(s-\tau(s, \xi)) \Delta \xi \Delta s \\
& +P(t) \int_{c}^{d} x(t-\tau(t, \xi)) \Delta \xi,
\end{aligned}
$$

where

$$
G(t, s)=\frac{e_{A}(s, t)}{e_{A}(\omega, 0)-1}
$$


Let $\mathbb{M}=\left\{x \in \Phi: m<x(t)<M, t \in[0, \omega]_{\mathbb{T}}\right\}$. We observe that $\mathbb{M}$ is a bounded, closed, and convex subset of $\Phi$. Define two mappings $\mathcal{A}, \mathcal{B}: \mathbb{M} \rightarrow \Phi$ as follows

$$
(\mathcal{A} x)(t)=\frac{1}{r(t)} \int_{t}^{t+\omega} G(t, s) \int_{c}^{d} f(s, x(s-\tau(s, \xi)))-Q(s) P^{\sigma}(s) x^{\sigma}(s-\tau(s, \xi)) \Delta \xi \Delta s,
$$

$$
(\mathcal{B} x)(t)=P(t) \int_{c}^{d} x(t-\tau(t, \xi)) \Delta \xi .
$$

For any $x \in \mathbb{M}$ and $t \in \mathbb{T}$, we have from (3.4) and (3.5) that

$$
\begin{aligned}
& (\mathcal{A} x)(t+\omega) \\
& =\frac{1}{r(t+\omega)} \int_{t+\omega}^{t+2 \omega} G(t, s) \int_{c}^{d} f(s, x(s-\tau(s, \xi)))-Q(s) P^{\sigma}(s) x^{\sigma}(s-\tau(s, \xi)) \Delta \xi \Delta s \\
& =\frac{1}{r(t)} \int_{t}^{t+\omega} G(t+\omega, v+\omega) \int_{c}^{d} f(v+\omega, x(v+\omega-\tau(v+\omega, \xi))) \\
& -Q(v+\omega) P^{\sigma}(v+\omega) x^{\sigma}(v+\omega-\tau(v+\omega, \xi)) \Delta \xi \Delta v \\
& =\frac{1}{r(t)} \int_{t}^{t+\omega} G(t, v) \int_{c}^{d} f(v, x(v-\tau(v, \xi)))-Q(v) P^{\sigma}(v) x^{\sigma}(v-\tau(v, \xi)) \Delta \xi \Delta v \\
& =(\mathcal{A} x)(t),
\end{aligned}
$$

and

$$
(\mathcal{B} x)(t+\omega)=P(t+\omega) \int_{c}^{d} x(t+\omega-\tau(t+\omega, \xi)) \Delta \xi=(\mathcal{B} x)(t),
$$

which means that $\mathcal{A} x$ and $\mathcal{B} x$ are $\omega$-periodic. For all $x, y \in \mathbb{M}$, and $t \in \mathbb{T}$, from (3.1), we get

$$
\begin{aligned}
& (\mathcal{A} x)(t)+(\mathcal{B} y)(t) \\
& =\frac{1}{r(t)} \int_{t}^{t+\omega} G(t, s) \int_{c}^{d} f(s, x(s-\tau(s, \xi)))-Q(s) P^{\sigma}(s) x^{\sigma}(s-\tau(s, \xi)) \Delta \xi \Delta s \\
& +P(t) \int_{c}^{d} y(t-\tau(t, \xi)) \Delta \xi \\
& \leq \frac{1}{r_{0}} \int_{t}^{t+\omega} G(t, s) A(s) \int_{c}^{d} r^{\sigma}(s)\left(\frac{f(s, x(s-\tau(s, \xi)))}{Q(s)}-P^{\sigma}(s) x^{\sigma}(s-\tau(s, \xi)) \Delta \xi\right) \Delta s \\
& +p_{1} M \\
& \leq\left(1-p_{1}\right) M+p_{1} M=M,
\end{aligned}
$$


and

$$
\begin{aligned}
& (\mathcal{A} x)(t)+(\mathcal{B} y)(t) \\
& =\frac{1}{r(t)} \int_{t}^{t+\omega} G(t, s) \int_{c}^{d} f(s, x(s-\tau(s, \xi)))-Q(s) P^{\sigma}(s) x^{\sigma}(s-\tau(s, \xi)) \Delta \xi \Delta s \\
& +P(t) \int_{c}^{d} y(t-\tau(t, \xi)) \Delta \xi \\
& \geq \frac{1}{r_{1}} \int_{t}^{t+\omega} G(t, s) A(s) \int_{c}^{d} r^{\sigma}(s)\left(\frac{f(s, x(s-\tau(s, \xi)))}{Q(s)}-P^{\sigma}(s) x^{\sigma}(s-\tau(s, \xi)) \Delta \xi\right) \Delta s \\
& \geq m,
\end{aligned}
$$

which give that $m \leq(\mathcal{A} x)(t)+(\mathcal{B} y)(t) \leq M$ for all $x, y \in \mathbb{M}$ and $t \in \mathbb{T}$. Thus $\mathcal{A} x+\mathcal{B} y \in \mathbb{M}$ for all $x, y \in \mathbb{M}$.

for all $x, y \in \mathbb{M}$, we have

$$
\begin{aligned}
& |(\mathcal{B} x)(t)-(\mathcal{B} y)(t)| \\
& =\left|P(t) \int_{c}^{d} x(t-\tau(t, \xi)) \Delta \xi-P(t) \int_{c}^{d} y(t-\tau(t, \xi)) \Delta \xi\right| \\
& \leq P(t) \int_{c}^{d}|x(t-\tau(t, \xi))-y(t-\tau(t, \xi))| \Delta \xi \\
& \leq p_{1}\|x-y\| .
\end{aligned}
$$

Hence $\mathcal{B}$ defines a contraction mapping with contraction constant $p_{1}$.

Next,we show that $\mathcal{A}$ is completely continuous. First, we shall show that $\mathcal{A}$ is continuous.

Let $\left\{x_{k}\right\} \in \mathbb{M}$ be a convergent sequence of functions such that $x_{k}(t) \rightarrow x(t)$ as $k \rightarrow \infty$. Because $\mathbb{M}$ is closed, for $x \in \mathbb{M}$. For $t \in[0, \omega]_{\mathbb{T}}$, we have

$$
\begin{aligned}
& \left|\left(\mathcal{A} x_{k}\right)(t)-(\mathcal{A} x)(t)\right| \\
& =\mid \frac{1}{r(t)} \int_{t}^{t+\omega} G(t, s) \int_{c}^{d} f\left(s, x_{k}(s-\tau(s, \xi))\right)-Q(s) P^{\sigma}(s) x_{k}^{\sigma}(s-\tau(s, \xi)) \Delta \xi \Delta s \\
& -\frac{1}{r(t)} \int_{t}^{t+\omega} G(t, s) \int_{c}^{d} f(s, x(s-\tau(s, \xi)))-Q(s) P^{\sigma}(s) x^{\sigma}(s-\tau(s, \xi)) \Delta \xi \Delta s \mid \\
& \leq \frac{1}{r_{0}} \int_{t}^{t+\omega} G(t, s) \int_{c}^{d}\left|f\left(s, x_{k}(s-\tau(s, \xi))\right)-f(s, x(s-\tau(s, \xi)))\right| \Delta \xi \Delta s \\
& +\frac{1}{r_{0}} \int_{t}^{t+\omega} G(t, s) Q(s) P^{\sigma}(s) \int_{c}^{d}\left|x_{k}^{\sigma}(s-\tau(s, \xi))-x^{\sigma}(s-\tau(s, \xi))\right| \Delta \xi \Delta s .
\end{aligned}
$$

Because

$$
\left|f\left(s, x_{k}(s-\tau(s, \xi))\right)-f(s, x(s-\tau(s, \xi)))\right| \rightarrow 0
$$


and

$$
\left|x_{k}^{\sigma}(s-\tau(s, \xi))-x^{\sigma}(s-\tau(s, \xi))\right| \rightarrow 0
$$

as $k \rightarrow \infty$ and $\xi \in[c, d]_{T_{T}}$, by making use of the Lebesgue dominated convergence theorem, we see that

$$
\lim _{k \rightarrow \infty}\left\|\mathcal{A} x_{k}-\mathcal{A} x\right\|=0
$$

This means that $\mathcal{A}$ is continuous. Second, we prove that $\mathcal{A} \mathbb{M}$ is relatively compact. It suffices to show that the family of functions $\{\mathcal{A} x: x \in \mathbb{M}\}$ is uniformly bounded and equicontinuous on $[0, \omega]_{\mathbb{T}}$. From $(3.4)$, we see that

$$
|(\mathcal{A} x)(t)|
$$

$$
\begin{aligned}
& =\sup _{t \in[0, \omega]_{\mathbb{T}}} \frac{1}{r(t)} \int_{t}^{t+\omega} G(t, s) \int_{c}^{d} f(s, x(s-\tau(s, \xi)))-Q(s) P^{\sigma}(s) x^{\sigma}(s-\tau(s, \xi)) \Delta \xi \Delta s \\
& \leq \frac{1}{r_{0}} \sup _{t \in[0, \omega]_{\mathbb{T}}}\left|\int_{t}^{t+\omega} G(t, s) A(s) \int_{c}^{d} r^{\sigma}(s)\left[\frac{f(s, x(s-\tau(s, \xi)))}{Q(s)}-P^{\sigma}(s) x^{\sigma}(s-\tau(s, \xi))\right] \Delta \xi \Delta s\right| \\
& \leq\left(1-p_{1}\right) M
\end{aligned}
$$

and

$$
\begin{aligned}
& \left|(\mathcal{A} x)^{\Delta}(t)\right| \\
& =\left|\left[\frac{1}{r(t)}\right]^{\Delta} r(t)(\mathcal{A} x)(t)+\frac{1}{r^{\sigma}(t)}\left[-Q(t) x^{\sigma}(t)+\int_{c}^{d} f(t, x(t-\tau(t, \xi))) \Delta \xi\right]\right| \\
& =\left|\left[-\frac{r^{\Delta}(t)}{r^{\sigma}(t) r(t)}\right] r(t)(\mathcal{A} x)(t)+\frac{1}{r^{\sigma}(t)}\left[-Q(t) x^{\sigma}(t)+\int_{c}^{d} f(t, x(t-\tau(t, \xi))) \Delta \xi\right]\right| \\
& \leq \frac{1}{r_{0}}\left\|r^{\Delta}\right\|\|\mathcal{A} x\|+\frac{\|Q\|}{r_{0}} M+\frac{\|Q\|}{r_{0}}\left[\frac{r_{0}\left(1-p_{1}\right) M}{r_{0}}+p_{1} M\right] \\
& \leq \frac{1}{r_{0}}\left\|r^{\Delta}\right\|\left(1-p_{1}\right) M+\frac{M}{r_{0}}\|Q\|+\frac{M}{r_{0}}\|Q\| \\
& \leq \frac{M}{r_{0}}\left[\left\|r^{\Delta}\right\|\left(1-p_{1}\right)+2\|Q\|\right],
\end{aligned}
$$

which give that $\{\mathcal{A} x: x \in \mathbb{M}\}$ is uniformly bounded and equicontinuous on $[0, \omega]_{\mathbb{T}}$. Therefore, $\mathcal{A} \mathbb{M}$ is relatively compact. By Theorem 3.1 there is an $z \in \mathbb{M}$ such that $\mathcal{A} z+\mathcal{B} z=z$. It is easy to see that $x$ is a positive $\omega$-periodic solution of (1.1). 
Theorem 3.3. Assume $-1<p_{0} \leq P(t)(d-c) \leq 0$ and there exist positive constants $m$ and $M$ with $0<m<M$ such that

$$
\frac{r_{1}\left(m-p_{0} M\right)}{d-c} \leq r^{\sigma}(t)\left[\frac{f(t, x)}{Q(t)}-P^{\sigma}(t) x^{\sigma}(t)\right] \leq \frac{r_{0} M}{d-c}, \quad \forall(t, x) \in[0, \omega]_{\mathbb{T}} \times[m, M]
$$

Then, (1.1) has a positive w-periodic solution $x \in[m, M]$.

Proof. The proof is similar to that of Theorem 3.2, so we give only a rough sketch of the proof. Let $\mathbb{M}, G, \mathcal{A}$ and $\mathcal{B}$ be as in the proof of Theorem 3.2. It is clear that $\mathcal{A} x$ and $\mathcal{B} x$ are $\omega$-periodic. For all $x, y \in \mathbb{M}$ and $t \in \mathbb{T}$, from (3.6) we get

$$
\begin{aligned}
& (\mathcal{A} x)(t)+(\mathcal{B} y)(t) \\
& =\frac{1}{r(t)} \int_{t}^{t+\omega} G(t, s) \int_{c}^{d} f(s, x(s-\tau(s, \xi)))-Q(s) P^{\sigma}(s) x^{\sigma}(s-\tau(s, \xi)) \Delta \xi \Delta s \\
& +P(t) \int_{c}^{d} y(t-\tau(t, \xi)) \Delta \xi \\
& \leq \frac{1}{r_{0}} \int_{t}^{t+\omega} G(t, s) A(s) \int_{c}^{d} r^{\sigma}(s)\left(\frac{f(s, x(s-\tau(s, \xi)))}{Q(s)}-P^{\sigma}(s) x^{\sigma}(s-\tau(s, \xi)) \Delta \xi\right) \Delta s \\
& \leq M
\end{aligned}
$$

and

$$
\begin{aligned}
& (\mathcal{A} x)(t)+(\mathcal{B} y)(t) \\
& =\frac{1}{r(t)} \int_{t}^{t+\omega} G(t, s) \int_{c}^{d} f(s, x(s-\tau(s, \xi)))-Q(s) P^{\sigma}(s) x^{\sigma}(s-\tau(s, \xi)) \Delta \xi \Delta s \\
& +P(t) \int_{c}^{d} y(t-\tau(t, \xi)) \Delta \xi \\
& \geq \frac{1}{r_{1}} \int_{t}^{t+\omega} G(t, s) A(s) \int_{c}^{d} r^{\sigma}(s)\left(\frac{f(s, x(s-\tau(s, \xi)))}{Q(s)}-P^{\sigma}(s) x^{\sigma}(s-\tau(s, \xi)) \Delta \xi\right) \Delta s \\
& +p_{0} M \\
& \geq\left(m-p_{0} M\right)+p_{0} M \geq m,
\end{aligned}
$$

which give that $m \leq(\mathcal{A} x)(t)+(\mathcal{B} y)(t) \leq M$ for all $x, y \in \mathbb{M}$ and $t \in \mathbb{T}$. Thus $\mathcal{A} x+\mathcal{B} y \in \mathbb{M}$ for all $x, y \in \mathbb{M}$. 
For all $x, y \in \mathbb{M}$, we have

$$
\begin{aligned}
& |(\mathcal{B} x)(t)-(\mathcal{B} y)(t)| \\
& =\left|P(t) \int_{c}^{d} x(t-\tau(t, \xi)) \Delta \xi-P(t) \int_{c}^{d} y(t-\tau(t, \xi)) \Delta \xi\right| \\
& \leq|P(t)| \int_{c}^{d}|x(t-\tau(t, \xi))-y(t-\tau(t, \xi))| \Delta \xi \\
& \leq\left|p_{0}\right| \| x-y|| .
\end{aligned}
$$

Hence $\mathcal{B}$ defines a contraction mapping with contraction constant $\left|p_{0}\right|$.

Next, we show that $\mathcal{A}$ is completely continuous. First, we shall show that $\mathcal{A}$ is continuous. The continuity of $\mathcal{A}$ can be shown as in proof Theorem 3.2. Second, we prove that $\mathcal{A M}$ is relatively compact. It suffices to show that the family of functions $\{\mathcal{A} x: x \in \mathbb{M}\}$ is uniformly bounded and equicontinuous on $[0, \omega]_{\mathbb{T}}$. From $(3.4)$, we see that

$|(\mathcal{A} x)(t)|$

$$
\begin{aligned}
& =\sup _{t \in[0, \omega]_{\mathbb{T}}}\left|\frac{1}{r(t)} \int_{t}^{t+\omega} G(t, s) \int_{c}^{d} f(s, x(s-\tau(s, \xi)))-Q(s) P^{\sigma}(s) x^{\sigma}(s-\tau(s, \xi)) \Delta \xi \Delta s\right| \\
& \leq \frac{1}{r_{0}} \sup _{t \in[0, \omega]_{\mathbb{T}}}\left|\int_{t}^{t+\omega} G(t, s) A(s) \int_{c}^{d} r^{\sigma}(s)\left[\frac{f(s, x(s-\tau(s, \xi)))}{Q(s)}-P^{\sigma}(s) x^{\sigma}(s-\tau(s, \xi))\right] \Delta \xi \Delta s\right| \\
& \leq M
\end{aligned}
$$

and

$$
\begin{aligned}
& \left|(\mathcal{A} x)^{\Delta}(t)\right| \\
& =\left|\left[\frac{1}{r(t)}\right]^{\Delta} r(t)(\mathcal{A} x)(t)+\frac{1}{r^{\sigma}(t)}\left[-Q(t) x^{\sigma}(t)+\int_{c}^{d} f(t, x(t-\tau(t, \xi))) \Delta \xi\right]\right| \\
& =\left|\left[-\frac{r^{\Delta}(t)}{r^{\sigma}(t) r(t)}\right] r(t)(\mathcal{A} x)(t)+\frac{1}{r^{\sigma}(t)}\left[-Q(t) x^{\sigma}(t)+\int_{c}^{d} f(t, x(t-\tau(t, \xi))) \Delta \xi\right]\right| \\
& \leq \frac{1}{r_{0}}\left\|r^{\Delta}\right\|\|\mathcal{A} x\|+\frac{\|Q\|}{r_{0}} M+\frac{\|Q\|}{r_{0}}\left[\frac{r_{0} M}{r_{0}}+\left|p_{0}\right| M\right] \\
& \leq \frac{1}{r_{0}}\left\|r^{\Delta}\right\| M+\frac{M}{r_{0}}\|Q\|+\frac{M}{r_{0}}\|Q\|\left(1+\left|p_{0}\right|\right) \\
& \leq \frac{M}{r_{0}}\left[\left\|r^{\Delta}\right\|+\|Q\|\left(2+\left|p_{0}\right|\right)\right]
\end{aligned}
$$


which give that $\{\mathcal{A} x: x \in \mathbb{M}\}$ is uniformly bounded and equicontinuous on $[0, \omega]_{\mathbb{T}}$. Therefore, $\mathcal{A} \mathbb{M}$ is relatively compact. By Theorem 3.1 there is an $z \in \mathbb{M}$ such that $\mathcal{A} z+\mathcal{B} z=z$. It is easy to see that $x$ is a positive $\omega$-periodic solution of (1.1).

Corollary 3.1. Assume $r(t)=1,0 \leq P(t)(d-c) \leq p_{1}<1$ and there exist positive constants $m$ and $M$ with $0<m<M$ such that

$$
\frac{m}{(d-c)} \leq\left[\frac{f(t, x)}{Q(t)}-P^{\sigma}(t) x^{\sigma}(t)\right] \leq \frac{\left(1-p_{1}\right) M}{(d-c)}, \quad \forall(t, x) \in[0, \omega]_{\mathbb{T}} \times[m, M] .
$$

Then, (1.1) has a positive $\omega$-periodic solution $x \in[m, M]$.

Corollary 3.2. Assume $r(t)=1,-1<p_{0} \leq P(t)(d-c) \leq 0$ and there exist positive constants $m$ and $M$ with $0<m<M$ such that

$$
\frac{\left(m-p_{0} M\right)}{d-c} \leq\left[\frac{f(t, x)}{Q(t)}-P^{\sigma}(t) x^{\sigma}(t)\right] \leq \frac{M}{d-c}, \quad \forall(t, x) \in[0, \omega]_{\mathbb{T}} \times[m, M] .
$$

Then, (1.1) has a positive w-periodic solution $x \in[m, M]$.

\section{REFERENCES}

[1] M. Adivar, Y. N. Raffoul, Existence of periodic solutions in totally nonlinear delay dynamic equations, Electron. J. Qual. Theory Differ. Equ. 2009(1) (2009), 1-20.

[2] A. Ardjouni, A. Djoudi, Existence of positive periodic solutions for nonlinear neutral dynamic equations with variable delay on a time scale, Malaya J. Mat. 2(1) (2013), 60-67.

[3] A. Ardjouni, A. Djoudi, Existence of periodic solutions for nonlinear neutral dynamic equations with functional delay on a time scale, Acta Univ. Palacki. Olomuc. Fac. Rerum Nat. Math. 52(1) (2013), 5-19.

[4] A. Ardjouni, A. Djoudi, Existence of periodic solutions for nonlinear neutral dynamic equations with variable delay on a time scale, Commun. Nonlinear Sci. Numer. Simul. 17 (2012), 3061-3069.

[5] A. Ardjouni, A. Djoudi, Periodic solutions in totally nonlinear dynamic equations with functional delay on a time scale, Rend. Sem. Mat. Univ. Politec. Torino 68(4) (2010), 349-359.

[6] L. Bi, M. Bohner, M. Fan, Periodic solutions of functional dynamic equations with infinite delay, Nonlinear Anal. 68 (2008), 1226-1245.

[7] M. Bohner, A. Peterson, Advances In Dynamic Equations On Time scales, Birkhäuser, Boston, 2003.

[8] M. Bohner, A. Peterson, Dynamic Equations On Time Scales, An Introduction With Applications, Birkhäuser, Boston, 2001.

[9] F. Bouchelaghem, A. Ardjouni, A. Djoudi, Existence of positive solutions of delay dynamic equations, Positivity 21 (2017), 1483-1493.

[10] T. Candan, Existence of positive periodic solutions of first-order neutral differential equations, Math. Meth. Appl. Sci. 40 (2017), 205-209.

[11] J. K. Hale, S.M. Verduyn Lunel, Introduction To Functional Differential Equations, Springer-Verlag, New York, 1993. 
[12] S. Hilger, Ein Ma $\beta$ kettenkalkül mit anwendung auf zentrumsmannigfaltigkeiten, Ph. D. thesis, Universität Würzburg, 1988.

[13] E. R. Kaufmann, Y. N. Raffoul, Periodicity and stability in neutral nonlinear dynamic equations with functional delay on a time scale, Electron. J. Differential Equations 2007(27) (2007), 1-12.

[14] E. R. Kaufmann and Y.N. Raffoul, Periodic solutions for a neutral nonlinear dynamical equation on a time scale, J. Math. Anal. Appl. 319 (2006) 315-325.

[15] D. R. Smart, Fixed Point Theorems, Cambridge Tracts in Mathematics, no. 66, Cambridge University Press, London-New York, 1974. 